\title{
PREJUICIO RELIGIOSO Y CONFLICTO SOCIAL EN UNA PEQUEÑA SOCIEDAD MEDITERRANEA: EL CASO DE MALlorCa
}

(1286-1435)

pau Cateura Bennasser

Universitat de les Illes Balears

(Palma de Mallorca)

\section{SUMARIO}

1. Introducción.- 2. La creación del Call y la época del Ceremonioso.- 3.

Asalto al Call y la cuestión de los conversos.

\section{INTRODUCCIÓN}

Datos numéricos, todavfa por contrastar, fija la población judía de la Corona de Aragón en unas 30.000 personas'. De ellas, aproximadamente un diez por ciento vivian en las Baleares, a juzgar por los recuentos fiscales de la época. En cualquier caso, la comunidad judía de Mallorca -en las demás islas su situación es prácticamente desconocida- posela una especificidad que no revelan las cifras anteriores. En efecto, si consideramos que la población de la isla no sobrepaso los $\mathbf{5 0 . 0 0 0 ~ h a b i t a n t e s ~ a ~ l o ~ l a r g o ~ d e l ~}$ siglo XIV, salvo en sus primeras décadas, hay que convenir en una influencia importante de los judios en el mundo económico, cultural y cientifico insular.

'Datos elaborados por J. RIERA y citados por D. Romano, en Els jueus en temps de Pere el Cerimoniós, De historia judía hispánica, Col.lecció Homenatges, Universitat de Barcelona Publicacions, 1991, pp. $460-461$.

"Anunio de Esoudios Modievales", 25 (1995) 
Acaso el inicio de la Baja Edad Media, en lo que a Mallorca y a la historia de los judios se refiere, pueda situarse en 1286. En esta fecha se propone el rey Alfonso III de Aragón la creación de un call o juderla en la capital de la isla, agrupando a todos los judios que vivian dispersos por la ciudad desde la época de la conquista2. En 1435, ante la amenaza de producirse un nuevo progrom, los iudios deciden su conversión y bautismo, aboliéndose definitivamente el call de Mallorca. Las dos fechas indicadas, en cuanto simbolizan respectivamente la crisis de la relación de cristianosjudíos y la eliminación de creencias disidentes, constituirán el umbral cronológico de nuestro trabajo, cuya pretensión es la de ofrecer una sintesis evolutiva de la cuestión judía a lo largo de este siglo y medio.

\section{2. la creación del Call y la época del Ceremonioso}

La conflictividad entre las Coronas de Aragón y de Mallorca llego a su punto álgido en el perfodo de 1284-1285. La alianza de Jaime II de Mallorca con la monarquia de los Capetos, como parte de un plan para sacudirse el vasallaje con respecto a los reyes de Aragón, registró el más absoluto fracaso. La invasión francesa de Cataluña no pudo alcanzar sus objetivos y el reino de Mallorca fue confiscado por Pedro III de Aragón e incorporado por su hijo Alfonso III a finales de 1285.

El cambio de dinastla fue la coyuntura aprovechada por ciertos estamentos de la ciudad para negociar con el rey, entre otros muchos temas, el del status y residencia de los judfos de la isla ${ }^{3}$. Como resultado, Alfonso III establecía que los judfos debran ser agrupados en un lugar de la ciudad y que no podrian ocupar cargos de relieve. Unos años después, en 1289, el mismo monarca establecla que los judfos no pudieran comprar

\footnotetext{
${ }^{2} R$. Soto CUMPANY, La aljama judaica de Ciutat en el siglo XII (Época de Jaime I), en el "Boletín de la Sociedad Arqueológica Luliana" (BSAL) (1978), pp. 145-184. Sobre los judíos de Ibiza, G. Mound, The Jews of Bbiza, en "Jerusalem Post" (if de marzo de 1983), p. 3, así como su comunicación inédita a las "Jomades sobre jueus i conversos de Balears a l"època del Descobriment d'Amèrica", Palma de Mallorca, 12-13 de mayo de 1992, con el título Criptojudios y manuscritos hebreos de Menorca, Bbiza y Formentera desde el siglo XIV al XVI.
}

3J. M. QUadrado, Privilegios y franquicias de Mallorca, Palma de Mallorea, 1895, I, pp. 68-69. Se trata de un privilegio capitulado de Alfonso III. 
bienes inmuebles 0 rentas ${ }^{4}$. Tales documentos nos revelan una comunidad judía económicamente floreciente, que inclura entre sus miembros a eficaces gestores, todo lo cual representaba una amenaza a ojos de los cristianos.

De acuerdo con los plazos previstos, en 1290 fue elegido por el procurador real el solar del call. Se trataba de una barriada en el extremo sudeste de la ciudad, zona evidentemente excéntrica, rodeada por la Casa del Temple, por el monasterio de las clarisas y por el convento de los franciscanos ${ }^{5}$. Se trataba de un arrabal no densamente poblado, en parte debido a la presencia molesta de tenerfas, y con espacios abiertos como los huertos del Temple y de un particular.

El traslado forzoso al nuevo call presentó problemas y contradicciones importantes. En la nueva zona de asentamiento, por las reticencias del monasterio de Santa Clara, no asi del Temple, a tener por vecinos a los judfos. Dicha institución se entregará por ello a una política de adquisiciones de inmuebles colindantes, creando entre el call y el monasterio una especie de barrera confesional. Mayor importancia revistió el proceso de venta de las casas poseidas por los judíos en la ciudad ${ }^{6}$, no exento de especulaciones en su contra, y de adquisiciones de viviendas y de solares en el nuevo call. Según la mencionada ordenanza real de 1289 , los judios no podian comprar bienes inmuebles. De mantener este criterio resultaba diffcil incentivar su establecimiento en el call, de ahf que el proceso de asentamiento se prolongara por lo menos hasta 1303 . En esta fecha, Jaime II de Mallorca les obligó a trasladar su residencia permanente al call, aunque permitiendo que siguieran conservando locales comerciales y artesanales fuera del mismo. También removió en parte la ordenanza de 1289, permitiéndoles la compra de fincas, pero sólo en el barrio judio.

El costoso proceso de traslado de los judíos fue en parte compensado, aunque indirectamente, por Jaime II al poner a su alcance una bolsa de

A. PONS, Los judios del reino de Mallorca durante los siglos. XIII-XIV, Palina de Mallorca, Miguel Font Editor, 1984, Vol. 2, pp. 210-211.

SJ. C. SASTRE, Santa Clara de Palına. Vida quotidiana en un inonestir medieval, Pulmu de Mallorca, Institut d'Estudis Balcàrics (IEB), 1993. Dicho monastcrio fuc fundado en 1256 sobre terrenos de Bemal de Sunta Eugenia.

'Un documento de la época señala que los judios hasla entonces consueverunt morari el suas domos el habilaciones habere insus Almudainam el in aliis locis civilatis, cosa lógica ya que fueron dotados, en tiempos de la conquista, tanto por el rey como por cl conde Nuño Sans. 
créditos derivada de su política de creación y reordenación de nuevas villas. El proposito real era el redistribuir la población de la isla en torno a unas dieciséis villas. Cada villa debra contar con cien pobladores, a quienes se distribufan lotes para construir casas, as como parcelas nisticas y otras aptas para el pastoreo'. Los gastos de instalación debfan correr a cuenta de los particulares interesados, limitándose la monarquia a fijar ciertas condiciones de los préstamos e intereses.

Durante todo el proceso de creación del call, la Iglesia de Mallorca habra desarrollado una intensa campaña. Es cierto que en 1300 el obispo, presionado por Jaime II, habla concedido autorización para construir una sinagoga, pero también lo es el desarrollo de una intensa campaña de proselitismo y algunos casos incluso de provocación. Sacerdotes que entraban en el barrio judio para administrar sacramentos o para predicar, y otros que provocaban alborotos contra los judios. En 1309, el rey orden 6 una investigación sobre los que habran propagado la acusación de que los judfos habłan matado a un niño cristiano. Por las misma fechas se haça tambien alusión a los contactos mantenidos por algunos conversos con judios.

Finalmente, en 1312 las acusaciones cristalizaron en la imputación a toda la comunidad judfa de Mallorca de haver convertido algunos cristianos al judarsmo'. En Cataluña, casos similares habían sido resueltos por el rey con una multa. En Mallorca, el rey Sancho tue convencido para aplicar medidas que, casi, suponfan la extinción de la aljama. En un primer momento decretó la confiscación de todos sus bienes muebles e inmuebles. Poco después decidió atenuar la medida, retornándoles los bienes necesarios para su sustento, pero a cambio de pagarle una multa de 95.000 libras (por motivos similares el rey de Aragón, en 1311, se habra limitado a imponer una multa de 500 libras a las aljamas de Cataluña) y aboliendo la sinagoga, que fue reconvertida en capilla católica. Por otra parte, el rey Sancho puso bajo su directo control a la aljama, al reservarse la designación de uno de sus secretarios.

\footnotetext{
'J. VICH y J. Munitani:k, Documenta regni Maioricarum (miscelánea), Palma de Mallorca, 1945, pp. 67-71. Dichas ordenanvas en cuanto suponen de reordenación del territorio han sido estudiadas por G. AloMAk. Uibanismo regional en la Edad Media: Les Ordinacions de Jaime II (I300) en el reino de Mallorca. Bureclona. Editorial Gustavo Gili, 1976.

A. PONS, Los judios, 2, pp. 218-219 y 226-227.

'G. Llompart y J. Rilira, La "Historia de la Sancta Fide Catholica" de Benet Espanyol (1548), cn "Fontcs Rerum Balcarium" (FRB), III (1979-1980), pp. 158-159.
} 
Durante este perfodo la comunidad judía de la isla estuvo en trance de desaparecer. Una nueva campaña de la Iglesia, con el bautizo de niños judios $^{10}$ y el compromiso arrancado, en 1323, a la monarquia de no retornar la sinagoga a la aljama trataban de quebrar su futuro. Procesiones de condenados a la horca trataban de aterrorizar. Sin embargo, los judios de la isla lograron liquidar la multa de 95.000 libras en 1327 e incluso pudieron prestar 5.000 libras al infante D. Felipe, tutor de Jaime III. A partir de esta época, la situación tendió a normalizarse $y$, a pesar de las resistencias eclesiásticas, obtener el placet real para construir una nueva sinagoga a partir de 1331.

En 1343, el reino de Mallorca fue reincorporado a la Corona de Aragón. Con la nueva dinastía, representada por Pedro el Ceremonioso, se restablecieron las relaciones tradicionales de la Corona con los judfos. Evidentemente esto suponfa, en las circunstancias concurrentes, una mejora de la situación de los judíos mallorquines ${ }^{11}$. Los primeros documentos otorgados por el rey de Aragón a su favor, en el periodo de 1343-1344, tratan de abarcar tres aspectos: el status jurídico y fiscal de la comunidad judía y la liberalización de sus actividades comerciales. En el primer aspecto confirmó todos los privilegios de la aljama, poniendo a la misma bajo su protección y salvaguardia. En el segundo aspecto, respetando la cuota de 165 libras anuales de contribución de la aljama. En cuanto al comercio, se rebajaban las tasas de tránsito de mercancías a los comerciantes judíos extranjeros y se suprimía la licencia del procurador real a los judios mallorquines que comerciaban en el exterior ${ }^{12}$.

Es correcta la afirmación de que la protección brindada por Pedro el Ceremonioso a los judíos mallorquines estaba guiada de motivos utilitarios, por los préstamos y subsidios que le podfa ofrecer la aljama. Pero debe tenerse en cuenta que este mismo monarca sometio al municipio de

\footnotetext{
${ }^{10} \mathrm{~A}$. Pons, Los judios, 2, p. 261. Es evidente que tanto la presión eclesiástica como la multa del rey tenían el objetivo coincidente de hacer desaparecer la aljama de Mallorca.

"Un panorama de la situación de los judios de la Corona de Aragón puede verse en D. Romuno, Els jueus en temps, pp. 455-473. Pero una perspectiva más general se encuentra en Y. BAER, Historia de los judíos en la Esparia cristiana, 2 vols., Madrid, Altalena, 1981. Para el caso interesa el vol. 2, pp. 324-377.

${ }^{12}$ Sobre el reinado de eate monarca P. CATEURA, Politica y finanzas del reino de Mallorca bajo Pedro IV de Aragón, Palma de Mallorea, IEB, 1982. El rey permitió a los judíos residir en edificios colindantes con el call, aunque extramuros.
} 
Mallorca a una importante presión financiera, hasta el punto de provocar su quiebra en 1372.

Por otra parte, la Iglesia de la isla perdio la influencia anterior, al tener que negociar con una monarquía mucho más poderosa que la privativa. Es cierto que existieron conflictos entre el obispo y el gobernador, pero más por temas de política fiscal ${ }^{13}$, es decir de la contribución del clero, que por la política confesional de la monarquia. Esto no significa que la Iglesia cediera en sus prácticas de proselitismo. Está documentada la utilización de conversos para misiones de predicación y de disputa con los judios, entre 1359 y 1361 . Años después, la aljama negociaba con el obispado el tema del bautismo de los judios. En 1373 se llego al acuerdo de fijar un plazo de tres dras entre la solicitud del bautismo y la recepción del sacramento ${ }^{14}$.

El mayor control de la situación por la monarquía aragonesa permite la expansión de los judfos por el interior de la isla. Durante el reinado de Pedro el Ceremonioso, grupos de judios -una o varias familias- se establecen en un total de siete poblaciones, aparte de Inca, donde ya existra una comunidad asentada por Jaime I en 1230. En suma, pues, a lo largo de la segunda mitad del siglo XIV se producen dos fenómenos de alguna forma contradictorios: una relativa expansión de la comunidad judfa por el interior de la isla, merced a la protección real, y la progresiva escisión de dicha comunidad debido a la aparición del fenómeno converso. El primer aspecto obedece a la inserción de las villas en la política financiera de la Corona $^{15}$. La comunidad campesina debra contribuir con un tercio de la carga global de Mallorca. El segundo aspecto, forma parte de una estrategia cuya primera fase serfa la división y en última instancia la erradicación de la comunidad judía.

\footnotetext{
${ }^{13}$ A. Santamarfa, El gobiemo de Olfo de Prócida. Una década de la historia de Mallorca (1365-1375), en "Hispania", 25 (1965), pp. 184-218 y 367-412. Del mismo autor: En tomo a la situación de los judios conversos de Mallorca en el siglo XV, en "BSAL", 31 (1953-1960), pp. 185-197.

${ }^{14 J . N . ~ H I L L G A R T H ~ y ~ J . ~ R O S S E L L, ~ T h e ~ L i b e r ~ c o m m u n i s ~ c u r i a e ~ o f ~ t h e ~ d i o c e s e ~ o f ~ M a l l o r c a ~}$ (1364-1375), Université de Montréal, Publ. de l'Institut d'Études Médiévales, 1989, p. 75, doc. $n^{\circ} 205$, y A. PONS, Los judios, 1, pp. 237-238 y 243-244.

isp. Cateura, Política y finanzas, pp. 150-170. Es fenómeno bien conocido que la presión impositiva durante el periodo de la dinastía privativa fue relativamente baja, en tanto se produce un in crescendo durante el periodo del Ceremonioso con las secuelas de endeudamiento de las villas.
} 


\section{Asalto al call y la cuestión de los conversos}

La toma del call de la capital de Mallorca, en 1391, como símbolo de la ruptura de la convivencia (cada vez más compleja a lo largo del siglo XIV) entre cristianos y judíos es tema bien conocido ${ }^{16}$. Por ello pasaremos brevemente los hechos para fijarnos especialmente en sus interpretaciones y en su entorno. El día dos de agosto de 1391 un grupo numeroso de campesinos a los que se unieron elementos ciudadanos, después de haber destruido el pequeño call de Inca, asaltó la judería de Palma, matando a unos trescientos individuos y saqueando sus casas. El resto pudo refugiarse en la ciudadela de la Almudaina, bajo protección del gobernador, donde permanecieron durante un mes.

La historiografia tradicional ha interpretado el asalto al call, como un mero mimetismo de movimientos similares producidos en la Penínsu$\mathrm{la}^{17}$. En segundo lugar ha inculpado exclusivamente a los campesinos foraneos de la responsabilidad de los hechos. La primera interpretación constituye un hábil argumento para exonerar en parte de responsabilidades al tejido social isleño, pero poco consistente con la realidad de los hechos ${ }^{18}$. El segundo argumento está teñido de aristocratismo. El asalto seŕa, según esto, de responsabilidad de las clases bajas o populares, principalmente campesinos. Ambas interpretaciones, al menos en la forma que están planteadas, caen por su propio peso. El antisemitismo tenfa una ya larga tradición en la isla a partir del momento en que se promueve la creación del call, de la ciudad en 1286 y se ponen en práctica tácticas de proselitismo e incluso de intimidación, como las antes mencionadas procesiones de condenados a la horca en tiempos de Jaime III. Por otra parte, las clases dirigentes de la isla no están exentas de prejuicios al promover que los judios no pudieran ocupar cargos importantes (offici de senyor), ni comprar bienes

\footnotetext{
${ }^{16} \mathrm{~J} . M^{*}$ QUADRADO, La juderia de Mallorca, publicado en "Muscu Balcar", IV (1887) y reeditado, bajo el mismo título, en Palma de Mallorca, 1967, con un prólogo-cstudio de J. MUNTANER. Aparte de las obras ya citadas, una revisión actual del tema del asalto al call de la ciudad se encuentra en J. F. LOPLiZ, La revolta de 1391. Efectivament crisi social, en cl "XIll Congreso de Historia de la Corona de Aragón. Comunicaciones I", Palına de Mallorea, 1989, pp. $111-123$.

${ }^{17}$ Dicha versión fue sostenida por V. MUT, Historia general del reino de Mallorca, 1650 (Reedic. Palma de Mallorca, 1981, vol. 3, pp. 319-320) y recogida por historiadores posteriores como A. PONS, Los judios, 2, pp. 162-172.

${ }^{18}$ P. CATEURA, Problemática de los conversos mallorquines a fines de la Edad Media, en "Les Illes Balears i América", Palma de Mallorca, IEB, 1, 1992, pp. 51-56.
} 
inmuebles. Es evidente que para estos grupos resultaba intolerable que los judios pudieran colocarse en un plano de igualdad o de superioridad sobre ellos.

En cualquier caso, los incidentes de 1391 deben situarse en el contexto de la época. La incorporación de Mallorca a la Corona de Aragon, en 1343, tiene entre otras consecuencias la de involucrar Mallorca en la costosa política mediterránea de la Corona. Resulta evidente que las islas fueron ocupadas militarmente en dicha fecha, pese a haberse pactado con un sector de sus estamentos dirigentes su rápida capitulación. Este modus expugnandi es decisivo para explicarnos la posición de la nueva dinastra en la isla. En efecto, el rey de Aragón gozará en ella de una posición de fuerza, sin parangón posible con los demás territorios de la Corona.

Durante años, Mallorca subvencionará, casi sin oposición, la política real, hasta sumirse en la bancarrota financiera. Es cierto que dicha crisis no sólo es achacable a la monarquía sino también a la coyuntura economica, y a los intereses y especulaciones que suscita la gestion del aparato financiero del municipio. Pero la conjunción de estos tres elementos determina el enquistamiento de la deuda pública y de un gravoso sistema impositivo destinado a alimentarla. Gravoso especialmente para los campesinos sobre los que recaen cuatro grupos de detracciones: las rentas y gravámenes señoriales, las rentas eclesiásticas (los diezmos), los impuestos generales de la isla y los derramados en cada pueblo por necesidades locales. Ante situaciones apremiantes los campesinos buscan una fugaz liquidez en la venta de censos consignativos, con lo que a menudo empeoran la rentabilidad de sus explotaciones ${ }^{19}$.

Si nos situamos nuevamente en 1391, el problema para los campesinos y también para los artesanos no era tanto la situación financiera mencionada como las nulas perspectivas de mejora. Los circulos dirigentes por el contrario, tratan de congelar dicha situación en beneficio suyo. La proliferación de impuestos les era beneficiosa al permitirles especular con el mercado de los arrendamientos de impuestos. Dado que a su vez eran acreedores de la deuda pública del municipio, se aseguraban el pago regular de sus inversiones, y dado el predominio absoluto, en el sistema fiscal vigente entonces, de los impuestos indirectos, soslayaban agresiones signifi-

\footnotetext{
${ }^{19} \mathrm{P}$. CATteuru, Fiscalidad real y municipal en la Mallorca del siglo XIV, en "Anuario de Estudios Medicvalcs", 22 (1922), pp. 443-462.
} 
cativas a su patrimonio personal. Este será el terreno fundamental donde se suscitará el conflicto entre el colectivo de campesinos-menestrales con los grupos dirigentes de la ciudad.

Este conflicto involucrará a los judíos, insertando un elemento irracional a sus reivindicaciones. Campesinos y menestrales perciben el status, de los judíos, pese a su exclusión de las áreas de poder, como formando parte del complejo económico-fiscal del reino. A menudo, a pesar suyo, los judíos participarán en operaciones financieras promovidas por la monarquia ${ }^{30}$, además de las especificas y tradicionales del crédito a particulares. Como que el préstamo era practicado también por cristianos, fue el factor religioso, es decir el resorte irracional e intolerante, el que hizo de los judíos objeto de la violencia desatada por dichos elementos populares. Pero llegados a este punto, cahe tener en cuenta dos aspectos de la cuestión: que en realidad los campesinos dicen y practican lo que el resto o al menos un sector importante de la sociedad piensa (la desaparición del judaísmo en la isla) y que la violencia de aquel año no sólo se dirigió contra los judíos sino también contra significados cristianos. Un grupo de éstos, básicamente cuadros responsables de la administración ciudadana, tuvieron que refugiarse en el castillo de Bellver, que fue asediado por los campesinos en octubre de $1391^{21}$.

Tras los hechos de este año, la situación era muy confusa. La sociedad judía resultó escindida en tres grupos: los que se convirtieron al cristianismo en un primer momento para evitar males mayores, los que huyeron en dirección al $\mathbf{N}$. de Africa y un tercer grupo formado por los que permanecieron en la isla y conservaron su religion y cultura, a los que se unieron en 1394-1395 un grupo de judíos portugueses ${ }^{22}$. De una sociedad dual, de cristianos y judíos, se pasa en esta época a una sociedad tripartita al incorporarse los conversos.

\footnotetext{
${ }^{20}$ En 1381 , el rey a cambio de ciertas prestaciones económicas de la aljama, consigna a esta una renta anual de $\mathbf{4 4 3}$ libras sobre escribanías y parroquias de la isla (ARM, Rp-33, fols. 78 r. -87 r.).

${ }^{21}$ J.F. LOPEZ, La revolta de 1391, pp. 111-113. Una de las primeras exigencias de los campesinos fue la abolición de los impuestos sobre el consumo. Las repercusiones mediterráneas de los asaltos a las juderías en D. ABULAFIA, Una comunità ebraica della Sicilia occidentale: Erice 1298-1304, "Archivio Storico per la Sicilia Orientale", II-III (1984), pp. 157-190, glosando la floreciente comunidad judía previos dichos acontecimientos.

25. Muntaner, Prólogo a La judería en Mallorca de J.M" QUAdRado, p. 17. Desconocemos de quien partió la iniciativa, aunque contó, desde el primer momento con el favor real.
} 
El primer grupo en reorganizarse fue el de la minoría judía, llegando a disponer de dos sinagogas aunque por la presión de los cristianos tuvieron que renunciar a una de ellas en $1420^{23}$. Más compleja fue la organización de los conversos. No podfan vivir en sus antiguas casas del call, pero tampoco eran aceptados por los cristianos convencidos de la falsedad de sus convicciones religiosas ${ }^{2 a}$. Fruto de estas alternativas fue su organización como comunidad separada, a través del sistema de cofradía.

Disponemos de los estatutos de la cofradía de conversos aprobados en 1404 y ampliados en $1410^{25}$, lo cual nos permite establecer su situación en estas fechas y especialmente su evolución organizativa. Tal como figura en el cuadro comparativo, que adjunto, en 1404 los conversos elaboran unos estatutos de urgencia destinados a organizar un sistema de ayuda mutua. La fórmula institucional escogida es la cofradía y almoina (limosna), aungue no se designa ningún tipo de invocación o patrocinio religioso. Los cargos de la asociación se reducen a un comité ejecutivo de cuatro diputados y una comisión de seis prohombres, encargados de revisar las cuentas presentadas por los diputados. El control público de la asociación de conversos se ejerce a través del local escogido para las reuniones -la iglesia de san Francisco- ${ }^{26}$ y por el preceptivo permiso del gobernador para celebrar las reuniones. La condición de ciudadanos bajo sospecha determina que la persecución ideologica contra los conversos no cesara en sus formas más brutales hasta 1409 , cuando el rey ordeno suspender los registros domiciliarios indiscriminados, salvo que mediara orden judi$\mathrm{cial}^{27}$.

Los conceptos de ayuda estatutarios que se establecen son la asistencia a enfermos e indigentes, exequias y enterramiento y préstamos sobre prendas, es decir, se acuerda prestar por el valor que tenga la prenda entregada; dichos conceptos se hacian extensivos a los no asociados. Tales

\footnotetext{
${ }^{23}$ G. LlomparT, y J. RIERA, La "Historia de la Sancta Fide", p. 168.

${ }^{24}$ En los documentos posteriores al asalto y en las primeras décadas del siglo XV se remarca su condición de convers y de cristid novell, aunque a partir de 1440 e incluso antes deja de consignarse dicha condición.

${ }^{25}$ E. FNARNES, Cofradia de conversos del judaismo, en "BSAL", 7 (1897-1898), pp. 408410, y ARM, AH, S-33, fols. 295 r. -300 r.

${ }^{26} \mathrm{Como}$ ya se ha indicado dicho convento era colindante con el antiguo call.

${ }^{27}$ A. PONS, Constitucions e ordinacions del regne de Mallorca, en "BSAL", 24 (19321933), pp. 402 y $420-421$.
} 
previsiones de ayuda debran ser cubiertas con las cuotas de los socios (cuota de ingreso y cuota ordinaria semanal, a liquidar los viernes). De acuerdo con lo precedente es posible realizar una aproximación al presupuesto anual de la cofradía de conversos. Si consideramos que el número de éstos ${ }^{28}$, constatados documentalmente en 1391, era de 183 individuos, cifra que efectivamente pudo alterarse hasta 1404, los ingresos de este año pudieron ascender a 915 sueldos de cuotas de entrada y 1.464 sueldos de cuotas ordinarias semanales, dando un total de 2.379 sueldos. Esta cifra, desde luego sólo resulta aplicable el primer año, ya que en los siguientes los ingresos se reducirían a las cuotas semanales y alguna que otra alta como cofrade.

Los estatutos reformados, que el rey Martín el Humano aprueba en 1410 , presentan puntos de contraste importantes con el documento anterior. Se trata de un reglamento mucho más completo y detallado. Se estructura como cofradía, bajo la invocación religiosa de san Miguel Arcángel. Además resultan destacables dos aspectos importantes:

a) Autonomía: Si en 1404 los conversos se encuentran bajo control y tutela de las autoridades, ahora disponen de casa propia y pueden reunirse libremente, sin la previa licencia del gobernador.

b) Prestacion de servicios: Los nuevos estatutos de 1410 amplían notoriamente los servicios prestados tanto a cofrades como a conversos no asociados y forasteros. A los conceptos de ayuda establecidos en 1404, añaden ahora las subvenciones para casarse, asistencia a bodas, asistencia a detenidos y rescate de cautivos. Este último extremo se justifica por las actividades mercantiles de un sector de los conversos ${ }^{29}$.

La cofradía organizada en 1410 adquiere un carácter más rígido y jerárquico. Se reglamenta el sistema electoral, las competencias de los cargos, su remuneración, el sistema de multas y de ejecución de morosos, y los supuestos de expulsion. Se mantiene el sistema colegiado de cuatro sobreposats (nombre prestado de los gremios artesanales). Un comité de seis notables o prohombres interviene, junto con los sobreposats en la aceptación de las solicitudes de ingreso y en los casos de propuestas de expulsión, revisando las rendiciones de cuentas. Una comisión más amplia

\footnotetext{
20J. M* QUADRADO, La juderla, pp. 80-86, aunque este mismo autor asegura que su número pudo ser mayor al atestiguado por las fuentes.

${ }^{20} \mathrm{El}$ mismo Julia d'Oaca, uno de los negociadores de los nuevos estatutos, era mercader (ARM, P-2466, fol. 79 r.).
} 
de diez prohombres asume la función de fiscalizar los regalos propuestos por los dirigentes de la cofradía. El manefle o agente ejecutivo y el enterrador cierran el arco de los cargos permanentes de la institución.

La relativa apertura hacia los conversos mallorquines coincide con un endurecimiento de la situación de los judíos. Tras el asalto al call de 1391, las autoridades se habran limitado a renovar normas de control, cardas en desuso en épocas anteriores ${ }^{30}$. La aparición de un fenómeno nuevo, los conversos, los convierte en sospechosos de influencia sobre estos últimos. La atracción de judfos extranjeros -portugueses en 1394-1395 y norteafricanos en 1416- trataba de romper dicha relación, al separarles factores como la lengua y el parentesco. En cualquier caso, las autoridades diseñan estrategias globales de aislamiento de los judios tanto en Castilla como en la Corona de Arago $\mathrm{n}^{31}$. La normativa extendida a Mallorca en 1413 se concretaba en tres puntos básicos:

a) Aislamiento residencial: renovación del call, rodeado de un muro y con una sola puerta, como residencia obligatoria de los judios.

b) Constriccion de derechos: prohibición de llevar armas, de vestir determinadas prendas, de comer y beber con cristianos y de ejercer profesiones como médico, barbero, abogado, sastre, repostero y arrendador de derechos.

c) Constricción de actividades: venta de alimentos a cristianos y realizar prácticas de proselitismo. Pero no podfan oponerse al proselitismo cristiano.

El último capítulo se dedica a la cuestión de los conversos procedentes del $\mathbf{N}$. de Africa. Se prohibe su entrada en Mallorca en base a la sospecha de que acudian a Berberfa para hacer que sus hijos regresaran al judarsmo ${ }^{32}$.

Con tales medidas, tal como indica Baer los "legisladores no se proponfan aniquilar físicamente a los judíos. Sólo deseaban convertirlo al

\footnotetext{
${ }^{30}$ En 1393 el gobernador establecía que los judíos debían llevar un signo distintivo y visible consistente en una rueda mitad roja, mitad amarilla.

${ }^{31}$ La legislación antijudía de eata epoca fue inspirada por S. Vicente Ferrer primero en Castilla y después en Aragón. Entre ectiembre de 1413 y enero de 1414 visitó la isla en gire de predicación. Durante su estancia se produjo la publicación del documento.
}

${ }^{3}$ A. LIONEL ISMACs, Els jueus de Mallorca, Mallorca, M. Font Editor, 1986 (Edición original de 1936). 
cristianismo a través de la servidumbre y de la explotación"33. Pese a ello, la comunidad judra de Mallorca logro una nueva etapa de expansión. Es conocida la actividad comercial y aseguradora de Astruch Xibili, entre 1403 y 1435, quien era a su vez rabino. Durante este perfodo no es infrecuente encontrarlo asociado a mercaderes conversos y judfos del $N$. de Africa $^{24}$. Cabe destacar a este respecto que en 1416, de acuerdo con los protocolos notariales de A. Contestr, siete de las nueve sociedades comerciales estaban participadas parcial o totalmente por conversos. Al año siguiente, de las cinco sociedades creadas. tres de ellas estahan integradas por conversos y judíos. Una de ellas estaha formada por catorce socios distribuidos en la siguiente forma: tres judfos -Astruch Xihilf, Samuel Fatzuati y Faratx ben Amin-, nueve conversos y dos cristianos".

En suma pues las normas constrictoras de 1413 determinan adaptaciones y reorientaciones de la actividad de los judfos. Precisamente la prosperidad de la comunidad y sus intensas relaciones profesionales con los conversos influirán en la puesta en marcha de un nuevo proceso. En 1435 , los judios fueron acusados de escarnio a la pasión de Cristo, al haber utilizado un esclavo musulmán para parodiar dicha representacion. La inculpación afectó al rahino Astruch Xihill y a tres judíos más. Ante una amenaza de una repetición de los sucesos de 1391. los judóos intentaron sin conseguirlo dispersarse. Los inculpados fueron condenados a muerte pero al decidir recihir el hautisno. les fue conmutada la pena. Siguios su ejemplo el resto de la comunidad formada por más de doscientos individuos ${ }^{26}$. Aquí termina la presencia judía en la isla, después de poco más de doscientos años de coexistencia con los cristianos. A partir de entonces sólo hay una comunidad cristiana, aunque escindida, ya que los conversos estaban excluidos de los ámbitos públicos -cargos de la administración- y de los privados -sus estrategias matrimoniales se proyectan casi exclusivamente dentro de la comunidad o con familias de conversos de Valencia y Barcelona.

${ }^{33}$ Y. BAER, Historia de los judios, 2, p. 440.

${ }^{34} \mathrm{P}$. MACAIRE, Majorque et le commerce intemational (1400-1450 environ), Lille, 1986, Pp. $81-91$.

${ }^{35}$ ARM, P-2465, fols. 23 v., 24 v., 25 r. -25 v., 28,48 v., 49 v., 96 r. 99 r. y 115.

${ }^{36} \mathrm{P}$. Piferrer y J.M* QLAdRAdo, Islas Baleares, Palma, 1969 (Recdición), p. 120. 


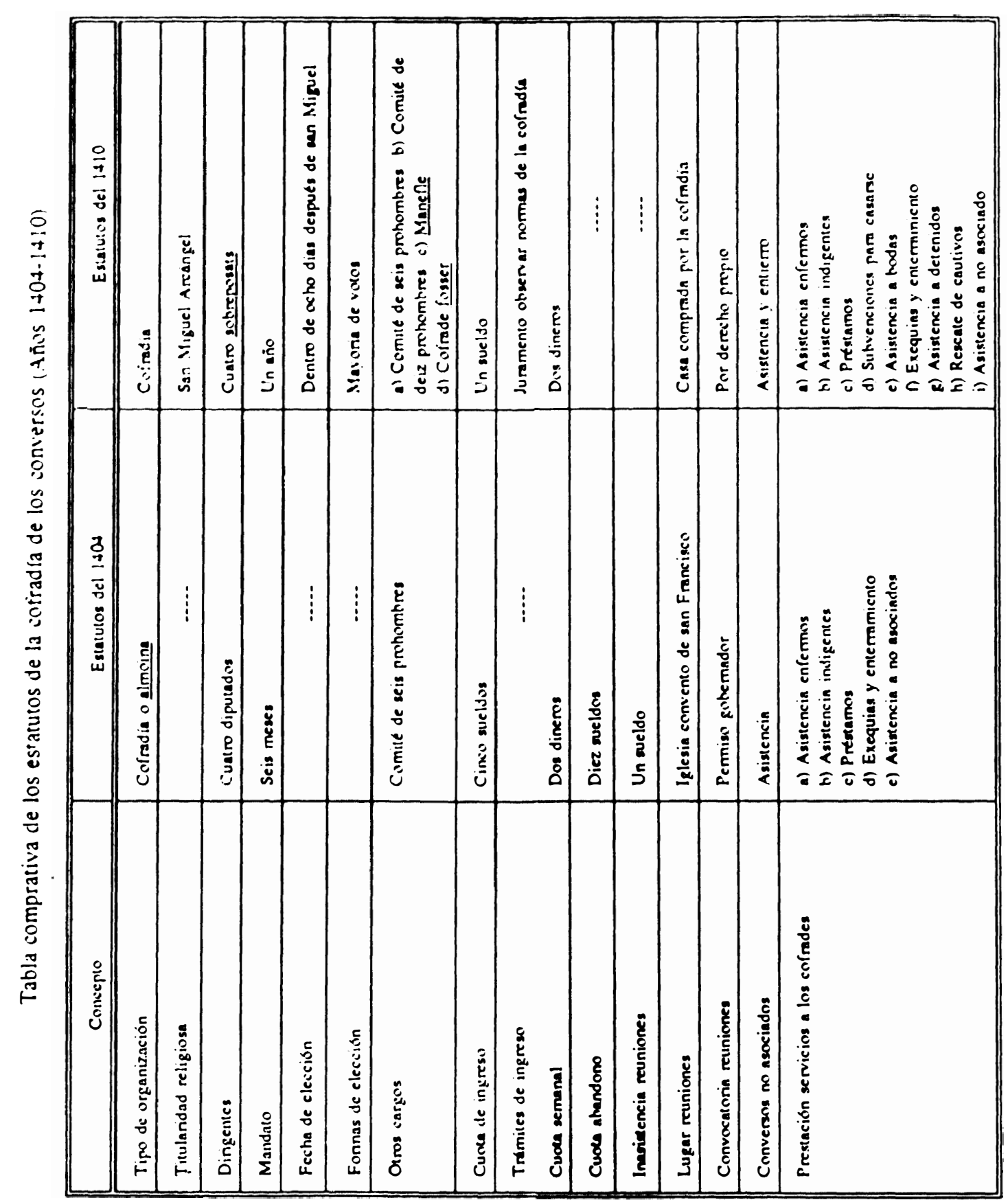




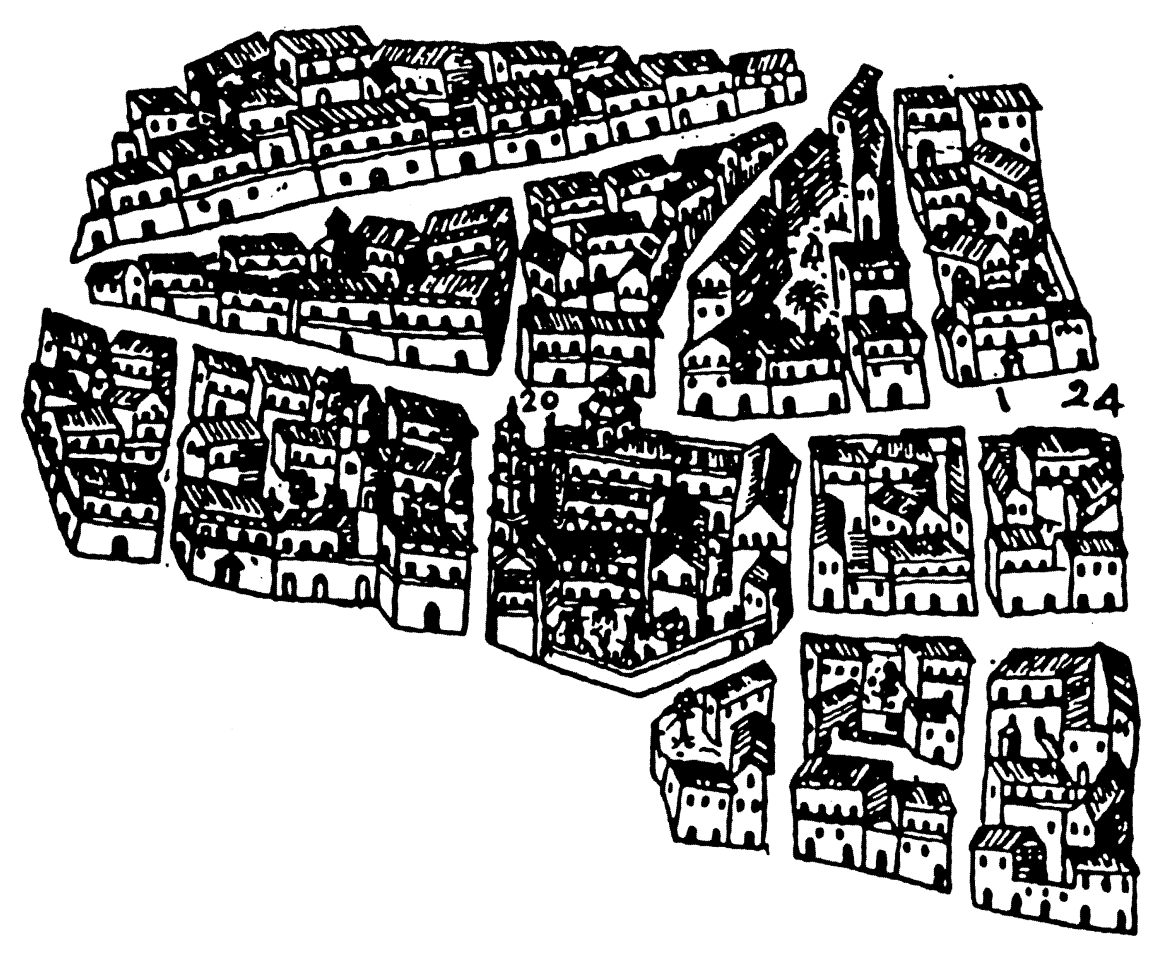

Plano del antiguo Call de la Ciudad de Mallorca (Antonio Garau, año 1644) 


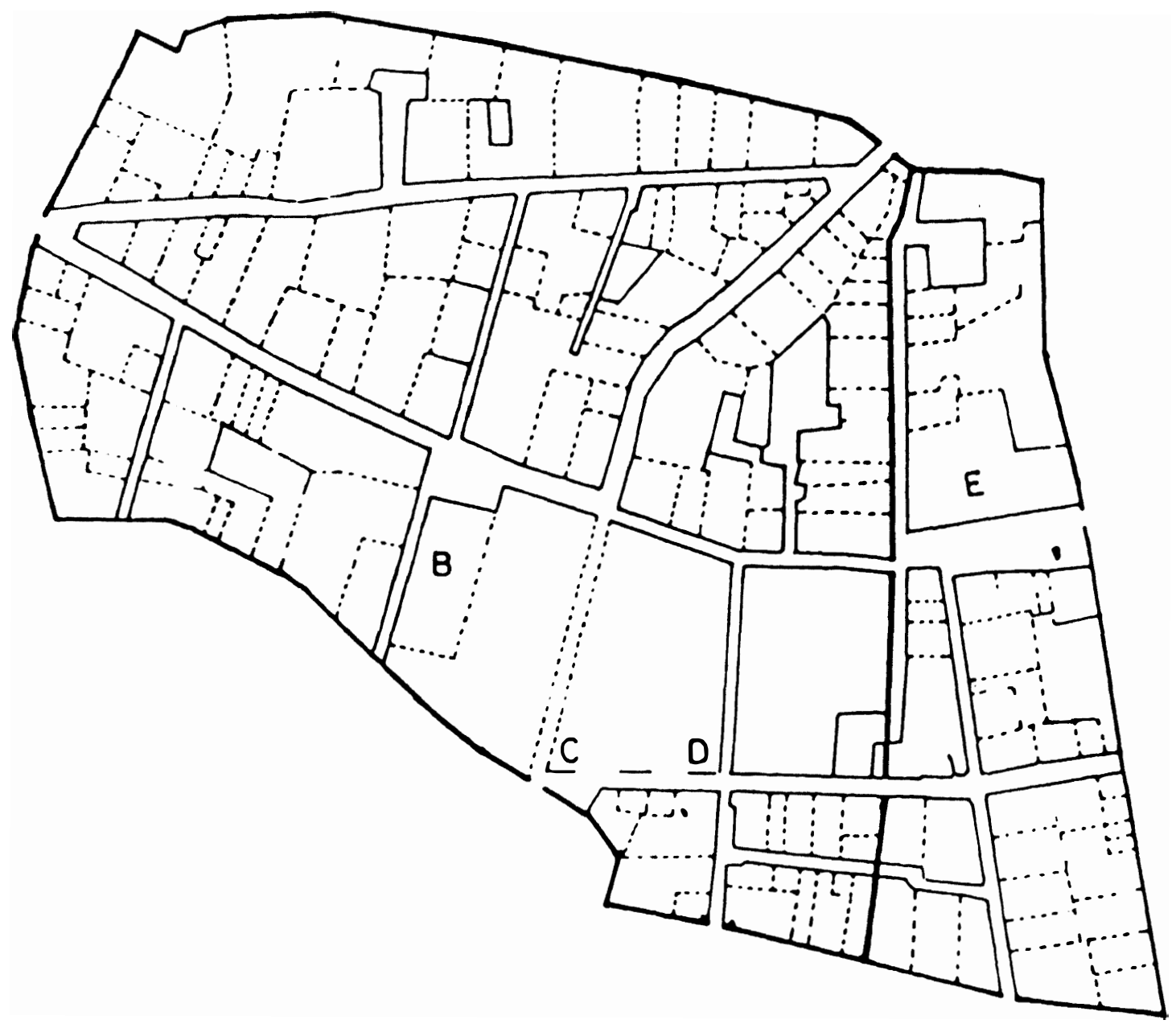

Reconstrucción del Call según Gabriel Alomar

(Historia de las islas Baleares, Palma de Mallorca, 1979, p. 194) 


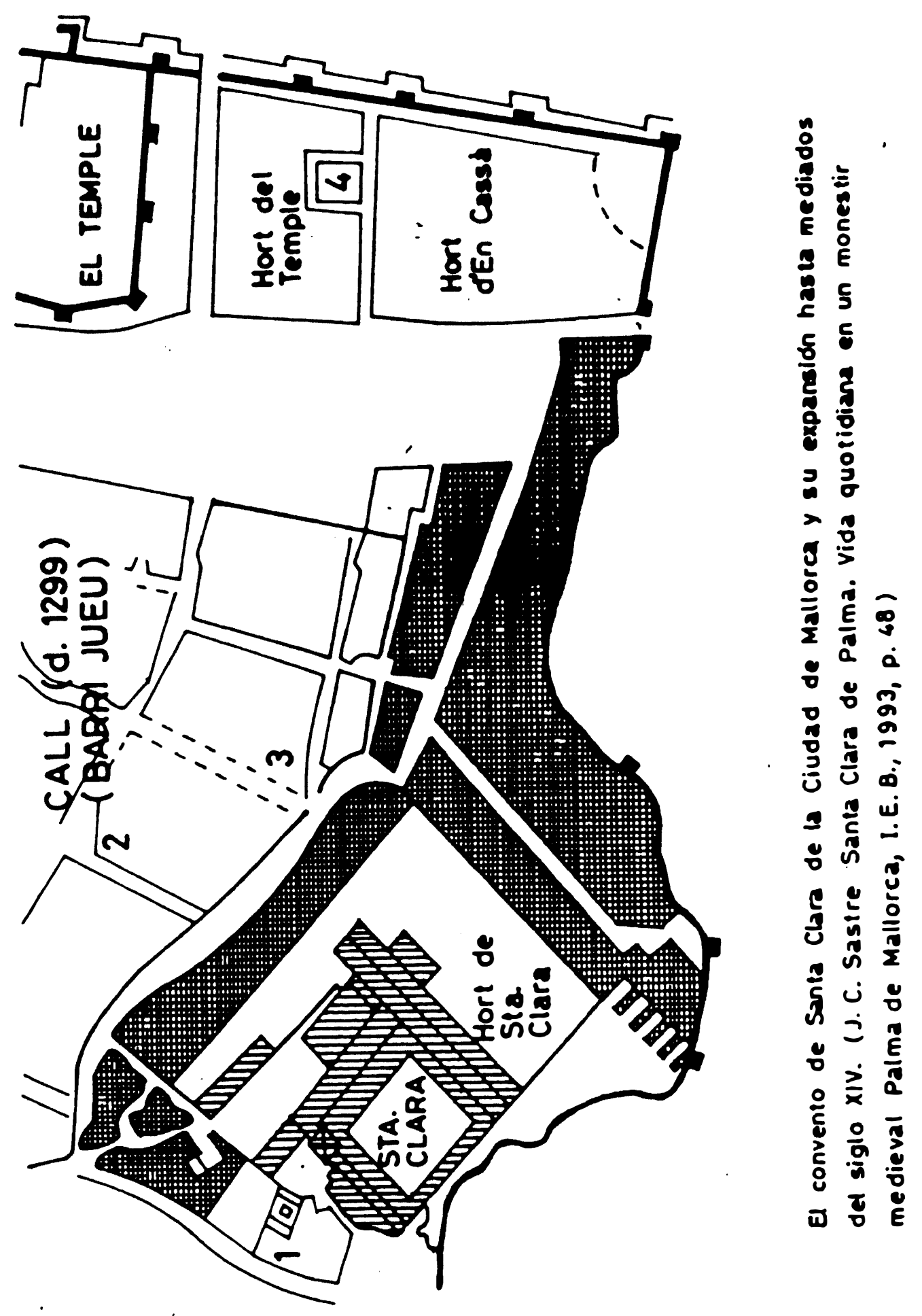




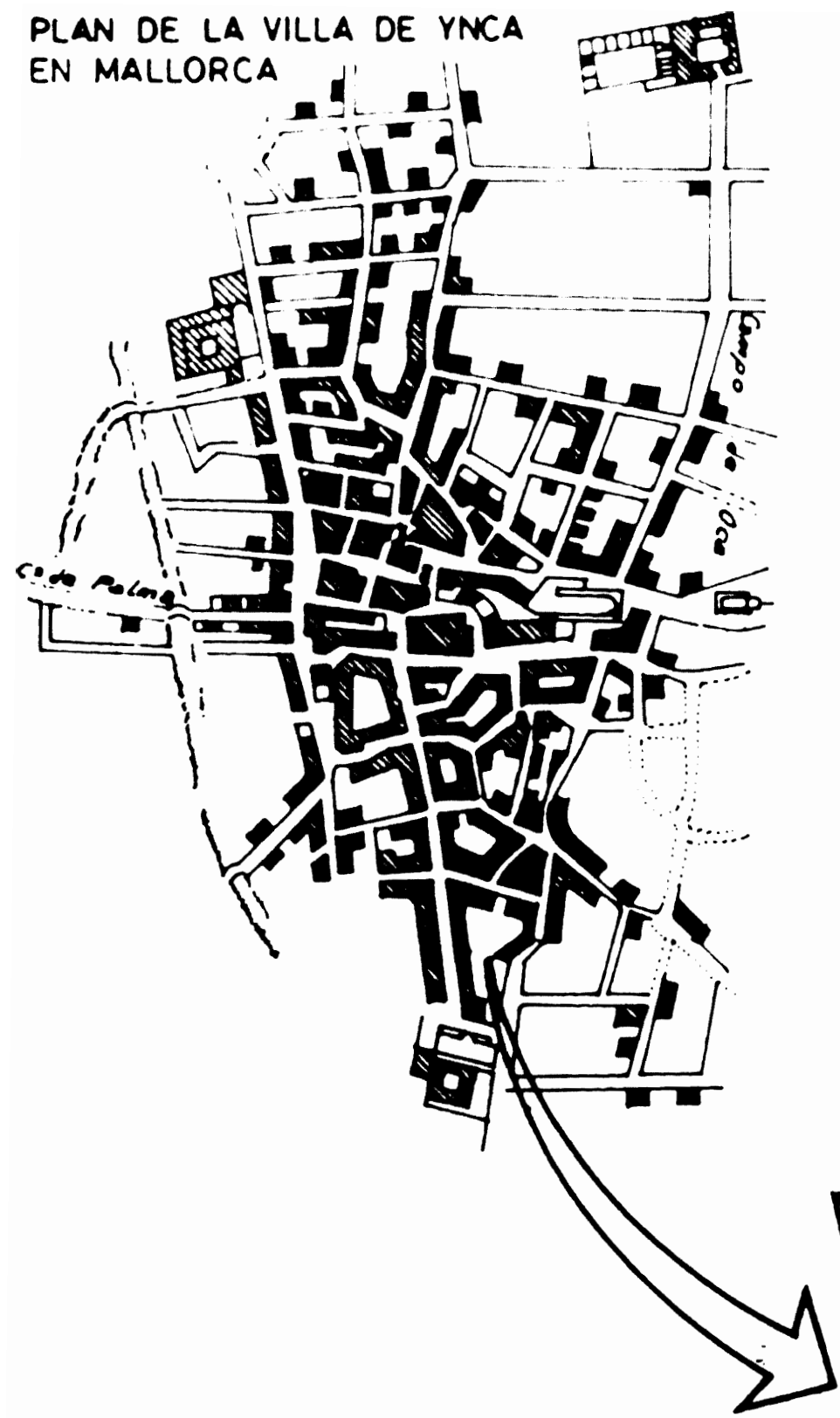

Plano general de la villa de inca levaniado en 1808 y ampliacion de la zona ocupada por a entiguo call (J. Barbert: Mda de sor Clara Andreu, Mallorea, 1007 )

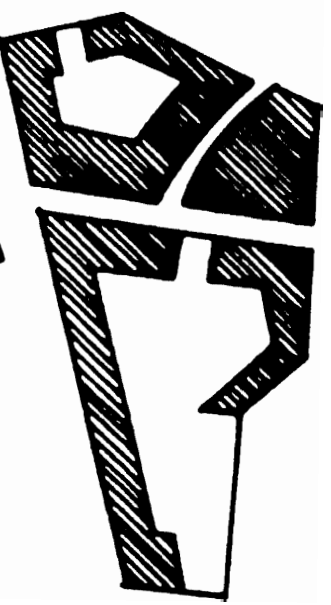




\section{RÉSUMÉ}

Dans cet article les traits distinctifs qui caractérisent l'évolution de la communáuté des Juifs de Majorque sont examinés.

Il s'agit d'une petite société méditerranéenne, à Majorque, pendant le Moyen Age.

Dans l'article, trois grandes phases chronologiques sont établies et on y étudie les changes de status de la communauté juive.

La première (1286-1315) est celle de la crtation du call ou judería (juiveric) dans la ville de Majorque ainsi que du procès entamé par le roi Sancho de Majorque contre la communauté et que finira par la confiscation de leurs proprićtés.

La deuxième, entre 1315 et 1391, contemple la problématiquc création d'une société juive dans l'lle depuis que le Royaume de Majorque est incorporé de nouveau a la Couronne d'Aragon, le 1343, par le roi Pierre le Cérémonicux, ainsi que l'insertion de la communauté juive dans l'appareil financier de la monarchie.

La troisième, entre 1391 et 1435 , met de relief la grave crise entre les communautés chrétienne et juive que finira avec l'assault au quartier juif de la ville de Majorque, ainsi que remarque l'apparition d'une nouvelle communauté des conversos, organisée très vite en une confrérie et la progressive dépersonnalisation des Juifs à cause de les mesures prises par les autorités et de l'atraction exercée par les Juifs d'origine portugais.

En tout cas, la consolidation d'une nouvelle communauté juive fut empêché par un nouveau procès en 1345 que détermina la conversion au Christianisme.

\section{SUMMARY}

The aim of this article is to examine the main features of the evolution of the Jewish Community in Majorca, a small Mediterranean society during the Middle Ages. In the article, three main phases are established, each one representing different moments of the stalus of the Majorcan Jews.

The first period (1286-1315) contemplates the foundation of the call or juderia (Jewish Quarter) in the city of Majorca as well as the pressure against the Jews exercised by king Sancho of Majorca, ending with the confiscation of their properties.

The second stage (1315-1391) contemplates the reconstruction of Majorcan Jewish society and the profound change in the relations between the Monarchy and the Jewish Community from the reinstatement of Majorca in the Crown of Aragon (1343) by the king Pedro el Ceremonioso, as well as the insertion of the Jews in the monarchy's financial machinery.

The third phase emphasizes the crisis between Christians and Jews that ends with the assault on the City's Jewish Quarter and contemplates also the appearance of a Converso community that organized itself very soon as a congregation, as the old Jewish Community was being progressively depersonalized by the atraction of Jews of portuguese origin and due to the pressure of the authorities.

Somehow, the consolidation of a new community was prevented by a process in 1435 which forced conversion and ended the Jewish Community in Majorca. 